\title{
EXTENSIONS OF MEASURES INVARIANT UNDER COUNTABLE GROUPS OF TRANSFORMATIONS
}

\author{
ADAM KRAWCZYK AND PIOTR ZAKRZEWSKI
}

\begin{abstract}
We consider countably additive, nonnegative, extended real-valued measures vanishing on singletons. Given a group $G$ of bijections of a set $X$ and a $G$-invariant measure $m$ on $X$ we ask whether there exists a proper $G$-invariant extension of $m$.

We prove, among others, that if $\mathbb{Q}$ is the group of rational translations of the reals, then there is no maximal $\mathbb{Q}$-invariant extension of the Lebesgue measure on $\mathbb{R}$. On the other hand, if $2^{\omega}$ is real-valued measurable, then there exists a maximal $\sigma$-finite $\mathbb{Q}$-invariant measure defined on a proper $\sigma$-algebra of subsets of $\mathbb{R}$.
\end{abstract}

\section{INTRODUCTION}

The fact that every nonuniversal measure on an arbitrary set $X$ has a proper extension is a well-known result of Loś and Marczewski [LM]. This paper is motivated by the following more general question, which we will refer to as the invariant extension problem:

Given a set $X$ and a group $G$ of bijections of $X$, does every nonuniversal, $G$-invariant measure on $X$ have a proper $G$-invariant extension? (cf. [P, p. 21]).

By a measure on $X$ we mean a countably additive, nonnegative, extended real-valued function defined on a $\sigma$-algebra $\mathfrak{M}$ of subsets of $X$, vanishing on all singletons, and assuming at least one positive finite value.

A measure $m$ on $X$ is called:

$\kappa$-additive if every union of less than $\kappa$ sets of measure 0 has measure 0 , where $\kappa$ is an uncountable cardinal;

finite if $m(X)<+\infty$;

$\sigma$-finite if $X$ is a countable union of sets of finite measure;

semiregular if every set of positive measure contains a subset of positive, finite measure;

complete if all subsets of sets of measure zero are measurable; universal if it is defined on $\mathscr{P}(X)$.

Received by the editors February 21, 1989 and, in revised form, May 16, 1989.

1980 Mathematics Subject Classification (1985 Revision). Primary 03E05; Secondary 28C10, 03 E55. 
Clearly, $X$ carries a universal measure if and only if the cardinality of $X$ is at least real-valued measurable. In this case we will say that $X$ is large. Otherwise, $X$ is small.

If $G$ is a group of bijections of $X$, a measure $m$ on a $\sigma$-algebra $\mathfrak{M}$ of subsets of $X$ is $G$-invariant if $g A \in \mathfrak{M}$ and $m(g A)=m(A)$ for any $g \in G$ and $A \in \mathfrak{M}$. Notice that the measure completion of a $G$-invariant measure is $G$-invariant, hence we always assume that measures under consideration are complete.

We are primarily concerned with $\sigma$-finite measures, postponing possible generalizations until the last section.

The following two cases of the invariant extension problem have been intensively investigaied:

Case 1. $X$ is the Euclidean space $\mathbb{R}^{n}$, and $G$ is a subgroup of the group $D_{n}$ of $X$ 's isometries.

Sierpiński (quoted in Szpilrajn [Sz]) asked whether every $D_{n}$-invariant extension of the Lebesgue measure $l_{n}$ on $\mathbb{R}^{n}$ has a proper $D_{n}$-invariant extension.

The final affirmative answer to this question was obtained by Ciesielski and Pelc [CP]. Using ideas of Hulanicki [Hu], they also proved that if $2^{\omega}$ is small, then every $\sigma$-finite $G$-invariant measure on $\mathbb{R}^{n}$ has a proper $G$-invariant extension. Further investigations of Ciesielski [C] and Zakrzewski [Z2] showed that if $m$ is a maximal $\sigma$-finite $G$-invariant measure on $\mathbb{R}^{n}$, then $m$ is concentrated on an affine subspace $L$ of $\mathbb{R}^{n}$ such that $L$ is closed under $G$ 's action and $|\{g \mid L: g \in G\}| \leq \omega$ (cf. [Z2, Theorem 2.3]). If, in addition, $m$ extends $l_{n}$, then $|G| \leq \omega$ (cf. [C, Corollary 3.1]).

These results essentially reduced the problem to the case of large continuum and at most countable $G$. The particularly intriguing question whether every nonuniversal, $\sigma$-finite, $\mathbb{Q}$-invariant measure on $\mathbb{R}$ (possibly extending $l_{1}$ ) has a proper $\mathbb{Q}$-invariant extension, $\mathbb{Q}$ being the group of all rational translations, was repeatedly stated by Harazišvili [Ha, Problem 4], Pelc [P, Problem 3.8], and Ciesielski [C, Problem 1].

Case 2. $X$ is a group, and $G$ is its subgroup. Each element $g \in G$ is identified with the associated left shift $\varphi_{g} x=g x$ for $x \in X$.

This case was studied by Pelc [P]. He proved that if $X$ is small, then the answer to the extension problem is always affirmative. The same is true if $X$ is commutative and $G$ is uncountable.

For countable $G$ 's the situation gets complicated. On one hand, if there exists a descending sequence $\left\langle G_{n}: n \in \omega\right\rangle$ of subgroups of $G$ such that $\bigcap_{n \in \omega} G_{n}=$ $\{e\}$ and each $G_{n}$ has finite index in $G$, then every nonuniversal $\sigma$-finite $G$ invariant measure on $X$ has a proper $G$-invariant extension. On the other hand, if $X$ is a group of measurable cardinality, and (the group isomorphic to) $\mathbb{Q}$ is a subgroup of $X$, then there exists a maximal nonuniversal finite $\mathbb{Q}$-invariant measure on $X$. 
Pelc posed a problem of finding a complete characterization of those countable subgroups $G$ of a given group $X$ for which the answer to the extension problem is positive (cf. [P, p. 29]).

The above review of results places in the center of interest the invariant extension problem for at most countable $G$ 's. The present paper is essentially devoted to its solution.

The role played in this case by the size of $X$ should be emphasized. If $X$ is small, then by a Hulanicki-style argument (see [Hu]), the answer to the problem is "yes" for any $G$. On the other hand, if $X$ is large, then there always exists a universal $\sigma$-finite $G$-invariant measure on $X$ (see [Z1]). At the same time, the only previously known example of a nonuniversal maximal $G$-invariant measure on a set $X$ required its cardinality to be at least measurable (see [P]).

The paper is organized as follows. In $\S 1$ we give a general solution to the extension problem for at most countable groups (Theorem 1.6). As a corollary we obtain an algebraic characterization of those at most countable subgroups $G$ of a given large group $X$, for which the answer is positive (Corollary 1.10). In $\S 2$ we restrict our attention to the case of $X=\mathbb{R}^{n}$ and $G$ being a subgroup of $D_{n}$. We give the following solution to the problem on extending $\mathbb{Q}$-invariant measures on $\mathbb{R}$ : if $\mathbb{R}$ is large, then there exists a maximal, nonuniversal, $\sigma$ finite, $\mathbb{Q}$-invariant measure on $\mathbb{R}$ (Corollary 2.5). On the other hand, there are no such measures among extensions of $l_{n}$ (Corollary 2.11). We prove that, in general, if $G$ is any subgroup of $D_{n}$, then every nonuniversal, $G$-invariant extension of $l_{n}$ has a proper $G$-invariant extension. This gives a strong affirmative answer to Sierpiński's question. $\S 3$ is devoted to generalizations of some results of $\S \S 1$ and 2.

We frequently use the following auxiliary fact, essentially due to Szpilrajn [Sz] (see also Pelc [P]).

0.1 . Lemma. Let $G$ be a group of bijections of a set $X$ and $m$ a semiregular $G$-invariant measure on $X$. If there exists a nonmeasurable subset $A \subset X$ such that $g A=A$ for every $g \in G$, then $m$ has a proper, semiregular, G-invariant extension.

We use standard set-theoretical notation and terminology. Ordinals are identified with sets of their predecessors and cardinals with initial ordinals. In particular, $\omega$ denotes the set of natural numbers and the first infinite cardinal. For a set $X, \mathscr{P}(X)$ denotes the power set of $X$, and $|X|$ the cardinality of $X$.

We use standard terminology to describe the action of a group $G$ on a set $X$. We say that $G$ acts on $X$ :

transitively if for every $x, y \in X$ there exists $g \in G$ such that $g x=y$; and freely if $f x=g x$ implies $f=g$ for any $f, g \in G$ and $x \in X$. A set $A \subset X$ is called $G$-invariant if $g A=A$ for every $g \in G$.

We use the following auxiliary notation:

$$
F x=\{f x: f \in F\} \text { - the } F \text {-orbit of } x, F \subseteq G, x \in X ;
$$


$F A=\bigcup\{F x: x \in A\}, F \subseteq G, A \subseteq X ;$

$\operatorname{fix}(g)=\{x \in X: g x=x\}, g \in G$;

$\operatorname{Fix}(G)=\bigcup\{f i x(g): g \in G, g \neq e\}$;

$e$-the neutral element of $G$,

$D_{n}$-the group of isometries of $\mathbb{R}^{n}$,

$l_{n}$-the Lebesgue measure on $\mathbb{R}^{n}$.

In $\S 2$ we use the fact that the group $D_{n}$ equipped with the topology of pointwise convergence is a topological group (cf., e.g., $[\mathrm{F}]$ ).

\section{INVARIANT MEASURES ON ARBITRARY SETS}

Throughout this section we assume that $G$ is an at most countable group of bijections of a set $X$.

Given a fixed selector $S$ of the family of $G$-orbits we introduce the following notation:

$$
\begin{gathered}
A^{(s)}=A \cap G s \text { for } A \subset X, s \in S, \\
\operatorname{supp}(A)=\left\{s \in S: A^{(s)} \neq \varnothing\right\}, \\
G_{s}=\{g \mid G s: g \in G\} \quad \text { for } s \in S, \quad \underline{G}=\prod_{s \in S} G_{s} .
\end{gathered}
$$

In the obvious way we identify $\underline{G}$ with the corresponding group of bijections of $X$.

Our goal is to solve the invariant extension problem for $G$ and $X$. By 0.1 , it suffices to focus attention on such measures that all $G$-invariant subsets of $X$ are measurable.

So let us investigate the properties of a given $\sigma$-finite $G$-invariant complete measure $m$ defined on a $\sigma$-algebra $\mathfrak{M} \subset \mathscr{P}(X)$ such that $G A \in \mathfrak{M}$ for every $A \subset S$.

\subsection{Lemma. The measure $m$ is $\underline{G}$-invariant.}

Proof. Take arbitrary $A \in \mathfrak{M}$ and $g \in \underline{G}$. By the definition of $\underline{G}$ and the countability of $G$, there exist $g_{0}, g_{1}, \ldots \in G$ and a partition $\left\{S_{n}: n \in \omega\right\}$ of $S$ such that $g\left|G S_{i}=g_{i}\right| G S_{i}$ for every $i \in \omega$. It follows that

$$
g A=\bigcup_{i \in \omega} g_{i}\left(A \cap G S_{i}\right),
$$

so $g A \in \mathfrak{M}$ and $m(g A)=m(A)$.

The crucial step in our analysis of $m$ is the following:

1.2. Lemma. Let $\varepsilon=\inf \{m(A): A \in \mathfrak{M}$ and $\operatorname{supp}(A)=S\}$. Then there exists a set $B \in \mathfrak{M}$ such that $\operatorname{supp}(B)=S, m(B)=\varepsilon$, and, moreover, for every $A \in \mathfrak{M}$ and $g \in G$

$$
m\left(\left\{s \in S:(g B \cap A)^{(s)} \neq \varnothing \text { and }(g B \backslash A)^{(s)} \neq \varnothing\right\}\right)=0 .
$$

Proof. First assume that $\varepsilon$ is finite. 
For each $n \in \omega$ take $A_{n} \in \mathfrak{M}$ such that $\operatorname{supp}\left(A_{n}\right)=S$ and $\lim _{n \rightarrow \infty} m\left(A_{n}\right)=$ $\varepsilon$; pick up $g_{n} \in \underline{G}$ such that $S \subset g_{n} A_{n}$. Set $B=\bigcap_{n \in \omega} g_{n} A_{n}$. Then, clearly, $\operatorname{supp}(B)=S$ and by $1.1, B \in \mathfrak{M}$ and $m(B)=\varepsilon$.

To prove the second part, take arbitrary $A \in \mathfrak{M}$ and $g \in G$. Set $S_{0}=\{s \in$ $S:(g B \cap A)^{(s)} \neq \varnothing$ and $\left.(g B \backslash A)^{(s)} \neq \varnothing\right\}$ and consider the sets

$$
B_{1}=\left(g B \cap G\left(S \backslash S_{0}\right)\right) \cup\left((g B \cap A) \cap G S_{0}\right)
$$

and

$$
B_{2}=\left(g B \cap G\left(S \backslash S_{0}\right)\right) \cup\left((g B \backslash A) \cap G S_{0}\right) .
$$

Then $B_{1}, B_{2} \in \mathfrak{M}, \operatorname{supp}\left(B_{1}\right)=\operatorname{supp}\left(B_{2}\right)=S$, and $B_{1} \cup B_{2}=B$. But $B_{1} \cap B_{2} \cap G S_{0}=\varnothing$, so $m\left(S_{0}\right)=0$ since, otherwise, either $m\left(B_{1}\right)$ or $m\left(B_{2}\right)$ would be less than $m(B)=\varepsilon$, contradicting the definition of $\varepsilon$.

If $\varepsilon=\infty$, then split $S$ into countably many pairwise disjoint $S_{n}$ 's, such that for every $n \in \omega$ the corresponding $\varepsilon_{n}$ is finite. Construct the $B_{n}$ 's as above. It is easy to see that $B=\bigcup_{n \in \omega} B_{n}$ does the job.

In what follows we shall need a familiar notion of a $G$-partition (see, e.g., [KaMe], where it is called "a partition into blocks relative to $G$ ").

1.3. Definition. Suppose $Y$ is a $G$-invariant subset of $X$, and $\mathbf{P}$ and $\mathbf{P}^{\prime}$ are partitions of $Y$. Then we have the following:

(a) $\mathbf{P}$ is a $G$-partition of $Y$ if $\forall A \in \mathbf{P} \forall g \in G \quad g A \in \mathbf{P}$.

(b) $\mathbf{P} \leq \mathbf{P}^{\prime}$ if $\forall A \in \mathbf{P} \quad \exists A^{\prime} \in \mathbf{P}^{\prime} \quad A \subset A^{\prime}$.

(c) $\mathbf{P} \leq_{\text {fin }} \mathbf{P}^{\prime}$ if $\mathbf{P} \leq \mathbf{P}^{\prime}$ and $\forall A^{\prime} \in \mathbf{P}^{\prime} \quad\left|\left\{A \in \mathbf{P}: A \subset A^{\prime}\right\}\right|<\omega$.

(d) $\mathbf{P}$ is trivial if $\mathbf{P}=\{\{y\}: y \in Y\}$.

(e) $\mathbf{P}$ is a minimal $G$-partition if it is $\leq_{\text {fin }}$-minimal among $G$-partitions.

(f) $\mathbf{P}^{(k)}=\{\bigcup W: W \in \mathscr{P}(\mathbf{P})$ and $|W|=k\}, k \leq \omega$.

If $Y$ is the $G$-orbit of $x \in X$, then the properties of $G$-partitions of $Y$ are reflected by the algebraic properties of $G$ and its subgroup st ${ }_{G}(x)=\{g \in$ $G: g x=x\}$, called the stabilizer of $x$ in $G$. This is due to the fact that $\mathbf{P}$ is a $G$-partition of $Y$ provided there exists a subgroup $H$ of $G$ containing st $_{G}(x)$ such that $\mathbf{P}=\{g H x: g \in G\}$ (see [KaMe]). In particular we have the following folklore-like result.

1.4. Proposition. Suppose that $x \in X$ and $\mathbf{P}$ is a G-partition of $G x$. Then the following are equivalent:

(i) $\mathbf{P}$ is nontrivial and minimal.

(ii) $\mathbf{P}=\{g H x: g \in G\}$ for a certain subgroup $H$ of $G$ properly containing $\mathbf{s t}_{G}(x)$ and such that every proper subgroup of $H$ containing $\mathbf{s t}_{G}(x)$ has infinite index in $H$.

Now the structure of $m$ can be completely described in terms of $G$-partitions of orbits. 
1.5. Theorem. Assume that $G$ is an at most countable group of bijections of $X$ and $m$ is a G-invariant $\sigma$-finite complete measure on $X$ such that $G A$ is measurable for every $A \subset X$. Let $S$ be a selection of $G$-orbits.

There exists a family $\left\{\mathbf{P}_{s}: s \in S\right\}, \mathbf{P}_{s}$ being a G-partition of Gs for each $s \in S$, such that:

$$
\mathfrak{M}=\left\{A \subset X: m\left(\left\{s \in S: A^{(s)} \notin \bigcup_{k \leq \omega} \mathbf{P}_{s}^{(k)}\right\}\right)=0\right\} .
$$

Moreover:

(i) If $\mathscr{S}$ is the union of a selector of the family $\left\{\mathbf{P}_{s}: s \in S\right\}$, then:

$$
m(A)=\sum_{k \leq \omega} k \cdot m\left(\mathscr{S} \cap G\left\{s \in S: A^{(s)} \in \mathbf{P}_{s}^{(k)}\right\}\right) \quad \text { for any } A \in \mathfrak{M} .
$$

(ii) If the measure is finite, then $m\left(\left\{s \in S:\left|\mathbf{P}_{s}\right|=\omega\right\}\right)=0$.

(iii) The measure is universal if and only if $m\left(\left\{s \in S: \mathbf{P}_{s}\right.\right.$ is nontrivial $\left.\}\right)=$ 0 .

(iv) The measure $m$ does not have any proper G-invariant extensions if and only if $m\left(\left\{s \in S: \mathbf{P}_{s}\right.\right.$ is not minimal $\left.\}\right)=0$.

(v) If a family $\left\{\mathbf{P}_{s}^{\prime}: s \in S\right\}$ of G-partitions satisfies (*), then $m\left(\left\{s \in S: \mathbf{P}_{s} \neq \mathbf{P}_{s}^{\prime}\right\}\right)=0$.

Proof. Let $\left\{g_{n}: n \in \omega\right\}$ be an enumeration of $G$. Take $\varepsilon$ and $B$ as in 1.2.

Set $S_{1}=\left\{s \in S:\left\{g_{n} B^{(s)}: n \in \omega\right\}\right.$ is not a $G$-partition $\}$ and define

$$
\mathbf{P}_{s}= \begin{cases}\left\{g_{n} B^{(s)}: n \in \omega\right\} & \text { if } s \in S \backslash S_{1}, \\ \{G s\} & \text { if } s \in S_{1} .\end{cases}
$$

We claim that $m\left(S_{1}\right)=0$. To see this, notice that $S_{1}=\bigcup_{n, m \in \omega} S_{n, m}$, where

$$
S_{n, m}=\left\{s \in S:\left(g_{n} B \cap g_{m} B\right)^{(s)} \neq \varnothing \text { and }\left(g_{n} B \backslash g_{m} B\right)^{(s)} \neq \varnothing\right\} \text {. }
$$

By $1.2, m\left(S_{n, m}\right)=0$ for every $n, m \in \omega$.

It follows that we can assume w.l.o.g. that $S_{1}=\varnothing$.

To prove the " $\subset$ " part of $(*)$, take an arbitrary $A \in \mathfrak{M}$. Notice that for each $s \in S, A^{(s)} \notin \bigcup_{k \leq \omega} \mathbf{P}_{s}^{(k)}$ if and only if there exists $n \in \omega$ such that $\left(g_{n} B \cap A\right)^{(s)} \neq \varnothing$ and $\left(g_{n} B \backslash A\right)^{(s)} \neq \varnothing$. Hence by $1.2, m\left(\left\{s \in S: A^{(s)} \notin\right.\right.$ $\left.\left.\bigcup_{k \leq \omega} \mathbf{P}_{s}^{(k)}\right\}\right)=0$.

To prove the " $\supset$ " part of $(*)$, take $A \subset X$ such that $m\left(\left\{s \in S: A^{(s)} \notin\right.\right.$ $\left.\left.\bigcup_{k \in \omega} \mathbf{P}_{s}^{(k)}\right\}\right)=0$. We can assume w.l.o.g. that $A^{(s)} \in \mathbf{P}_{s}$ for every $s \in \operatorname{supp}(A)$, since $A$ is equal to the union of countably many sets of this form and a measure zero set. But then, by the definition of $\mathbf{P}_{s}$, there exists $g \in \underline{G}$ such that $A=g(B \cap G \operatorname{supp}(A))$. By $1.1, A \in \mathfrak{M}$.

To prove (i), use $(*)$ and the $\underline{G}$-invariance of $m$. Note that $B$ as above is a possible $\mathscr{S}$. 
Point (ii) follows immediately from (i).

To prove (iii), notice that $m$ is universal if and only if $S \in \mathfrak{M}$. Then use $(*)$.

The proof of (iv) is broken into two parts.

" $\Rightarrow$ " Set $S_{2}=\left\{s \in S: \mathbf{P}_{s}\right.$ is not minimal $\}$ and suppose that $S_{2}$ is not a measure zero set. For each $s \in S_{2}$ fix a $G$-partition $\mathbf{Q}_{s}<_{\text {fin }} \mathbf{P}_{s}$. Thinning out $S_{2}$ if necessary, we can assume that there is $n>1$ such that $\mid\left\{A^{\prime} \in \mathbf{Q}_{s}: A^{\prime} \subset\right.$ $A\} \mid=n$ for any $s \in S_{2}$ and $A \in \mathbf{P}_{s}$.

Put

$$
\begin{aligned}
\mathfrak{M}^{\prime}=\{A \subset X & : A \cap G\left(S \backslash S_{2}\right) \in \mathfrak{M} \\
& \text { and } \left.m\left(\left\{s \in S_{2}: A^{(s)} \notin \bigcup_{k \leq \omega} \mathbf{Q}_{s}^{(k)}\right\}\right)=0\right\},
\end{aligned}
$$

and define a measure $m^{\prime}$ on $\mathfrak{M}^{\prime}$ by

$$
\begin{aligned}
m^{\prime}(A)= & m\left(A \cap G\left(S \backslash S_{2}\right)\right) \\
& +n^{-1} \sum_{k \leq \omega} k \cdot m\left(\mathscr{S} \cap G\left\{s \in S_{2}: A^{(s)} \in \mathbf{Q}_{s}^{(k)}\right\}\right) .
\end{aligned}
$$

It is easy to see that $m^{\prime}$ is a proper $G$-invariant extension of $m$, contrary to our assumption.

" $\Leftarrow$ " Let $m^{\prime}: \mathfrak{M}^{\prime} \rightarrow[0, \infty]$ be a $G$-invariant extension of $m$. We prove that $\mathfrak{M}=\mathfrak{M}^{\prime}$.

First notice that for any $Z \subseteq X$, if $m^{\prime}(Z)=0$, then $m(G Z)=0$, hence by completeness of $m, m(Z)=0$.

By what has already been proved, for every $s \in S$ there exists a $G$-partition $\mathbf{P}_{s}^{\prime}$ of $G s$ such that:

$$
A \in \mathfrak{M}^{\prime} \quad \text { if and only if } m^{\prime}\left(\left\{s \in S: A^{(s)} \notin \bigcup_{k \leq \omega} \mathbf{P}_{s}^{(k)}\right\}\right)=0
$$

for any $A \subset X$.

Since $\mathfrak{M} \subset \mathfrak{M}^{\prime}$, applying $(* *)$ to $A=\mathscr{S}$ we get $m\left(\left\{s \in S:\right.\right.$ not $\mathbf{P}_{s}^{\prime} \leq_{\text {fin }}$ $\left.\left.\mathbf{P}_{s}\right)\right\}=0$. But by the assumption, $m\left(\left\{s \in S: \mathbf{P}_{s}^{\prime}<_{\text {fin }} \mathbf{P}_{s}\right\}\right)=0$, so $m(\{s \in S:$ $\left.\left.\mathbf{P}_{s}^{\prime} \neq \mathbf{P}_{s}\right\}\right)=0$. In view of $(* *)$, this clearly forces $\mathfrak{M}=\mathfrak{M}^{\prime}$.

Finally, the uniqueness of the family $\left\{\mathbf{P}_{s}: s \in S\right\}$, as described in (v), follows easily from $(*)$.

Now we are ready to state the main result of this section.

1.6. Theorem. Assume that $G$ is an at most countable group of bijections of $X$. Then the following are equivalent:

(i) Every $\sigma$-finite (finite) nonuniversal G-invariant measure on $X$ has a proper G-invariant extension. 
(ii) The set $\{x \in X$ : there exists a (finite) nontrivial minimal G-partition of $G x\}$ is small.

(iii) The set $\{x \in X$ : there exists a subgroup $H$ of $G$ (of finite index in $G)$ properly containing $\mathrm{st}_{G}(x)$ and such that every proper subgroup of $H$ containing $\mathrm{st}_{G}(x)$ has infinite index in $\left.H\right\}$ is small.

Proof. We prove the $\sigma$-finite version. To get the finite one, argue as below using 1.5 (ii), when necessary.

(i) $\Rightarrow$ (ii) Suppose not (ii). There is $n \leq \omega$ and a large subset $S_{0}$ of $S$ such that for each $s \in S_{0}$ there exists an $n$-element, minimal, nontrivial $G$-partition $\mathbf{P}_{s}$ of $G s$. Let $m^{\prime}$ be a universal measure on $S$ such that $m^{\prime}(S)=m^{\prime}\left(S_{0}\right)=1$. Set

$$
\mathfrak{M}=\left\{A \subset X: m^{\prime}\left(\left\{s \in S_{0}: A^{(s)} \in \bigcup_{k \leq \omega} \mathbf{P}_{s}^{(k)}\right\}\right)=1\right\},
$$

and define a measure $m$ on $\mathfrak{M}$ by

$$
m(A)=\sum_{k \leq n} k \cdot m^{\prime}\left(\left\{s \in S: A^{(s)} \in \mathbf{P}_{s}^{(k)}\right\}\right) \quad \text { for } A \in \mathfrak{M},
$$

where $\omega \cdot r$ is understood to be 0 if $r=0$ and $\infty$ if $r>0$.

It is easy to see that $m$ is a $\sigma$-finite $G$-invariant measure on $X$ and $G A \in \mathfrak{M}$ for every $A \subset S$. By $1.5, m$ is nonuniversal and has no proper $G$-invariant extension, contradicting (i).

(ii) $\Rightarrow$ (i) Take a $\sigma$-finite, nonuniversal, $G$-invariant measure $m$ defined on a $\sigma$-algebra $\mathfrak{M} \subset \mathscr{P}(X)$. We want to find a proper $G$-invariant extension of $m$.

If there exists a set $A \subset S$ such that $G A \notin \mathfrak{M}$, then we are done by 0.1 .

If not, then $m$ satisfies the hypothesis of 1.5 .

So, let $\left\{\mathbf{P}_{s}: s \in S\right\}$ be the family of partitions determined by $m$ and let $\mathscr{S}$ be the union of a selector of this family. Define $m^{\prime}: \mathscr{P}(S) \rightarrow[0, \infty]$ by $m^{\prime}(A)=m(\mathscr{S} \cap G A)$ for $A \subset S$. It is easy to see that $m^{\prime}$ is a universal $\sigma$-finite measure on $S$. Hence, (ii) implies that if $S^{\prime}=\left\{s \in S: \mathbf{P}_{s}\right.$ is nontrivial and minimal $\}$, then $m\left(S^{\prime}\right)=m^{\prime}\left(S^{\prime}\right)=0$. But by $1.5\left(\right.$ iii), the set $\left\{s \in S: \mathbf{P}_{s}\right.$ is nontrivial $\}$ is not of $m$ measure 0 . So $\left\{s \in S: \mathbf{P}_{s}\right.$ is not minimal $\}$ is not of $m$ measure 0 , and we are done by 1.5 (iv).

(ii) $\Leftrightarrow$ (iii) This follows immediately from 1.4.

From 1.5 we can abstract a purely algebraic property of $G$ which appears to be connected with the problem of extending $G$-invariant measures.

We say that $G$ is indicable by finite groups if every nontrivial subgroup of $G$ has a proper subgroup of finite index in $G$. This terminology is derived essentially from that of Burns and Hale $[\mathrm{BH}]$.

From 1.4 we get

1.7. Proposition. Suppose that $x \in X$ and $G$ acts freely on $G x$. Then the following are equivalent: 
(i) There is no nontrivial minimal G-partition of $G x$.

(ii) $G$ is indicable by finite groups.

Let us recall that

$$
\operatorname{Fix}(G)=\{x \in X: g(x)=x \text { for some } g \in G, g \neq e\} .
$$

Notice that $G$ acts freely on $G x$ if and only if $x \notin \operatorname{Fix}(G)$.

Combining 1.6 with 1.7 we obtain

1.8. Corollary. If $G$ is indicable by finite groups, then every $\sigma$-finite nonuniversal $G$-invariant measure on $X$ such that $m(\operatorname{Fix}(G))=0$ has a proper $G$ invariant extension.

The above corollary admits a partial converse.

1.9. Corollary. Assume that the set $X \backslash \operatorname{Fix}(G)$ is large. If every $\sigma$-finite nonuniversal $G$-invariant measure on $X$ has a proper $G$-invariant extension, then $G$ is indicable by finite groups.

Proof. Use 1.7 and implication (i) $\Rightarrow$ (ii) of 1.6 .

The conjunction of 1.8 and 1.9 yields

1.10. Corollary. Suppose that $X$ is large and an at most countable $G$ acts freely on every $G$-orbit. Then the following are equivalent:

(i) Every $\sigma$-finite nonuniversal G-invariant measure on $X$ has a proper $G$-invariant extension.

(ii) $G$ is indicable by finite groups.

The hypotheses of 1.10 are satisfied if $X$ is a large group and $G$ is a countable subgroup of $X$ acting on $X$ by left shifts. Then 1.10 gives a solution to a problem of Pelc quoted in $\S 0$ (cf. [P, p. 29]). In order to compare it with Pelc's partial result (see $\S 0$ ), let us make the following observation.

1.11. Proposition. The following are equivalent:

(i) $G$ is indicable by finite groups.

(ii) For a certain $\eta<\omega_{1}$ there exists a descending sequence $\left\langle G_{\alpha}: \alpha \in \eta\right\rangle$ of subgroups of $G$ such that:

(1) $G_{0}=G$,

(2) $G_{\lambda}=\bigcap_{\alpha<\lambda} G_{\alpha}$ for limit $\lambda<\eta$,

(3) $\bigcap_{\alpha<\eta} G_{\alpha}=\{e\}$,

(4) $G_{\alpha+1}$ has finite index in $G_{\alpha}$ for each $\alpha<\eta$.

We close this section with an important corollary of 1.2 which will be useful later. 
1.12. Lemma. Suppose that $m$ is a $\sigma$-finite, G-invariant measure defined on a $\sigma$-algebra $\mathfrak{M} \subset \mathscr{P}(X)$.

If $\inf \{m(A): A \in \mathfrak{M}$ and $\forall x \in X \quad A \cap G x \neq \varnothing\}=0$, then $m$ has a proper $G$-invariant extension.

Proof. By 0.1 , it suffices to prove that there exists a $G$-invariant nonmeasurable set. Suppose otherwise. Then $m$ satisfies the hypotheses of 1.2. Accordingly, there exists a set $B \in \mathfrak{M}$ such that $B \cap G x \neq \varnothing$ for every $x \in X$ and $m(B)=0$. But $X=G B$ and $|G| \leq \omega$, so $m(X)=0$, a contradiction.

\section{INVARIANT MEASURES ON EUCLIDEAN SPACES}

Throughout this section $G$ denotes a subgroup of $D_{n}$, the group of all isometries of $\mathbb{R}^{n}$.

The results of $\S 1$ combined with those of [Z2] yield the following solution to the invariant extension problem for $G$ and $\mathbb{R}^{n}$.

2.1. Theorem. Assume that $G$ is an arbitrary subgroup of $D_{n}$. Then the following are equivalent:

(i) Every $\sigma$-finite nonuniversal G-invariant measure on $\mathbb{R}^{n}$ has a proper $G$-invariant extension.

(ii) The set $\left\{x \in \mathbb{R}^{n}:|G x| \leq \omega\right.$ and there exists a nontrivial minimal $G$-partition of $G x\}$ is small.

Proof. Set $L=\left\{x \in \mathbb{R}^{n}:|G x| \leq \omega\right\}$ and $G^{\prime}=\{g \mid L: g \in G\}$. Notice that $L$ is a $G$-invariant affine subspace of $\mathbb{R}^{n}$ and $G^{\prime}$ is a countable group of its isometries. It is also clear that $G$ - and $G^{\prime}$-partitions of $G x$ coincide for any $x \in L$.

Moreover, by the result of [Z2], the existence of a maximal $\sigma$-finite $G$ invariant measure on $\mathbb{R}^{n}$ is equivalent to the existence of a maximal $\sigma$-finite $G^{\prime}$-invariant measure on $L$. In view of the above remarks, the desired equivalence follows immediately from 1.6.

In order to obtain more information about the particularly important case of $G$ being countable, we should come back to the discussion following 1.6. As a consequence of 1.9 we get

2.2. Corollary. Assume that $|G| \leq \omega$ and $2^{\omega}$ is large. If every $\sigma$-finite nonuniversal $G$-invariant measure on $\mathbb{R}^{n}$ has a proper G-invariant extension, then $G$ is indicable by finite groups.

Proof. It suffices to notice that $\operatorname{Fix}(G)$ is the union of countably many proper affine subspaces of $\mathbb{R}^{n}$, so the set $\mathbb{R}^{n} \backslash \operatorname{Fix}(G)$ is large if $2^{\omega}$ is.

It is not difficult to see that for $n<3$ the converse to the above is also true.

2.3. Proposition. Assume that $|G| \leq \omega$ and $n=1,2$. If $G$ is a group of isometries of $\mathbb{R}^{n}$ indicable by finite groups, then every $\sigma$-finite nonuniversal $G$ invariant measure on $\mathbb{R}^{n}$ has a proper G-invariant extension. 
It is tempting to conjecture that the above is true for all $n$. Unfortunately, Strojnowski [St] found a counterexample showing that this is actually false for all $n>2$.

There is, however, an interesting family of groups, for which the conclusion of 2.3 holds for all $n$. Following [F] we say that $G$ is crystallographic if $G$ is discrete and the group $D_{n} / G$ is compact (remember that $D_{n}$ is a topological group-see $\S 0$ ).

It is not very hard to prove by algebraic methods that every crystallographic group is indicable by finite groups and, moreover, the following is true.

2.4. Proposition. If $G$ is crystallographic, then every $\sigma$-finite nonuniversal $G$ invariant measure on $\mathbb{R}^{n}$ has a proper G-invariant extension.

We close the discussion of the extension problem for arbitrary $G$-invariant measures on $\mathbb{R}^{n}$ with the explicit statement of the solution to Pelc's problem on $\mathbb{Q}$-invariant measures (cf. [P, Problem 3.8]).

2.5. Corollary. If $2^{\omega}$ is large, then there exists a maximal, nonuniversal, finite, $\mathbb{Q}$-invariant measure on $\mathbb{R}$.

It seems interesting to compare the last result with the following:

2.6. Proposition. If $b$ is an arbitrary irrational number, then every finite nonuniversal measure on $\mathbb{R}$, invariant with respect to $\mathbb{Q}$ and $b$, has a proper $\mathbb{Q}$ invariant extension.

Proof. Let $G$ be the subgroup of $D_{1}$ generated by $\mathbb{Q}$ and $b$, and let $m$ be a finite $G$-invariant measure on a $\sigma$-field $\mathfrak{M} \subset \mathscr{P}(\mathbb{R})$. Consider the measure $m_{1}$ on $\mathbb{R} / \mathbb{Q}$ defined by $m_{1}=m(\bigcup \mathscr{A})$ for every $\mathscr{A} \subset \mathbb{R} / \mathbb{Q}$ such that $\bigcup \mathscr{A} \in \mathfrak{M}$.

It is easy to see that $m_{1}$ is $G / \mathbb{Q}$-invariant. Clearly, $|G / \mathbb{Q}|=\omega$, so $m_{1}$ cannot be universal (cf. [P, Proposition 2.3]). It follows that there exists $A \subset \mathbb{R}$ such that $\mathbb{Q}+A \notin \mathfrak{M}$, so we are done by 0.1 .

From now on we turn our attention to extensions of the Lebesgue measure $l_{n}$ on $\mathbb{R}^{n}$. Our first goal is to find a solution to the invariant extension problem restricted to such measures.

We begin with stating a result of Ciesielski (see [C, Corollary 3.1]).

2.7. Proposition (Ciesielski). If $|G|>\omega$, then every G-invariant extension of $l_{n}$ has a proper $G$-invariant extension.

The following folklore-like lemma establishes connections between topological properties of $G$ and its orbits; $G$ is considered as a subspace of the topological group $D_{n}$ (see $\S 0$ ). We shall use the well-known fact that every subgroup of a topological Hausdorff group is either discrete or has no isolated points; moreover, every discrete subgroup is closed (see [HR]).

2.8. Lemma. (i) If $G$ is discrete, then every G-orbit is closed.

(ii) If $G$ is not discrete, then the $G$-orbit of every $x \in \mathbb{R}^{n} \backslash \operatorname{Fix}(G)$ is dense in itself and its closure has at most countably many connected components. 
Proof. Notice that:

(1) For any $x \in \mathbb{R}^{n}$ the closure of the $G$-orbit of $x$ is equal to the $\operatorname{Cl}(G)$ orbit of $x$, where $\mathrm{Cl}$ is the closure operation (in $D_{n}$ ).

(2) For any $x \in \mathbb{R}^{n} \backslash \operatorname{Fix}(G)$ the function $g \mapsto g x$ is a homeomorphism of $G$ onto $G x$.

Now everything is clear except from the last part of (ii). To prove the latter consider $O=G x$. By $(1), \mathrm{Cl}(O)=\mathrm{Cl}(G) x$, so $\mathrm{Cl}(O)$ is a continuous image of $\mathrm{Cl}(G)$. Hence it suffices to show that $\mathrm{Cl}(G)$ has at most countably many connected components. One way to see this is to treat $D_{n}$ as a Lie group and use the well-known theorem that every closed subgroup of a Lie group is a Lie group itself (cf., e.g., [War, Theorem 3.2]). ${ }^{1}$

2.9. Lemma. If $|G| \leq \omega$ and $G$ is not discrete, then every $G$-invariant extension of $l_{n}$ has a proper $G$-invariant extension.

Proof. Let $m$ be a $G$-invariant extension of $l_{n}$ defined on a $\sigma$-algebra $\mathfrak{M}$. By 1.12 , it suffices to show that $\inf \left\{m(A): A \in \mathfrak{M}\right.$ and $\left.\left(\forall x \in \mathbb{R}^{n}\right) \quad A \cap G x \neq \varnothing\right\}=$ 0 .

So fix an arbitrary $\varepsilon>0$ and find an open set $A$ with the following properties:

(1) $\operatorname{Fix}(G) \subset A$;

(2) $L_{q, i} \subset A$ for every $q \in \mathbb{Q}$ and $i \in\{1, \ldots, n\}$, where $L_{q, i}=\left\{x \in \mathbb{R}^{n}\right.$ : $\left.x_{i}=q\right\}$

(3) $l_{n}(A)<\varepsilon$.

We claim that $A \cap G x \neq \varnothing$ for each $x \in \mathbb{R}^{n}$. To see this, take an arbitrary $x \in \mathbb{R}^{n}$. If $x \in \operatorname{Fix}(G)$, then by (1), $x \in A$.

If $x \notin \operatorname{Fix}(G)$, set $O=G x$. By 2.8(ii), there is a connected subset $C$ of $\mathrm{Cl}(O)$ such that $|C|>1$. Then, by (2), $C \cap A \neq \varnothing$ and so $O \cap A \neq \varnothing$.

Now we are ready to state the solution to the invariant extension problem for extensions of the Lebesgue measure. It may be viewed as another strong negative answer to Sierpiński's problem quoted in $\S 0$.

2.10. Theorem. Every nonuniversal G-invariant extension of $l_{n}$ has a proper G-invariant extension.

Proof. If $|G|>\omega$, use 2.7. If $|G| \leq \omega$ and $G$ is not discrete, use 2.9 .

So assume that $G$ is discrete. Consider the partition $\mathbf{P}$ of $\mathbb{R}^{n}$ into $G$ orbits. By $2.8(\mathrm{i}), \mathbf{P}$ consists of closed subsets of $\mathbb{R}^{n}$. Furthermore, for any open $U \subset \mathbb{R}^{n}$ the set $\bigcup\{\mathscr{O} \in \mathbf{P}: \mathscr{O} \cap U \neq \varnothing\}=G U$ is open. Hence, by a wellknown theorem of Kuratowski and Maitra [KM], there exists a Borel selector $S$ of $\mathbf{P}$.

Now suppose that $m: \mathfrak{M} \rightarrow[0, \infty]$ is an arbitrary nonuniversal $G$-invariant extension of $l_{n}$.

\footnotetext{
${ }^{1}$ This argument was suggested to us by W. Szczechla.
} 
Let $m_{1}$ be the restriction of $m$ to the $\sigma$-field $\mathfrak{M}_{1}=\mathfrak{M} \cap \mathscr{P}(S)$. By a classical theorem of Los and Marczewski [LM], there exists a proper extension $m_{2}$ of $m_{1}$ defined on a $\sigma$-algebra $\mathfrak{M}_{2} \subset \mathscr{P}(S)$ such that $\mathfrak{M}_{1} \subset \mathfrak{M}_{2}$.

Define a $\sigma$-field $\overline{\mathfrak{M}}$ of subsets of $\mathbb{R}^{n}$ and a measure $\bar{m}$ on $\overline{\mathfrak{M}}$ by

$$
\overline{\mathfrak{M}}=\left\{A \subset \mathbb{R}^{n}: g[A] \cap S \in \mathfrak{M}_{2} \text { for every } g \in G\right\}
$$

and

$$
\bar{m}(A)=\sum_{g \in G} m_{2}(S \cap g A) \quad \text { for } A \in \overline{\mathfrak{M}} .
$$

Using the fact that $m(\operatorname{Fix}(G))=l_{n}(\operatorname{Fix}(G))=0$, it is easy to check that $\bar{m}$ is a proper $G$-invariant extension of $m$.

As an immediate corollary we explicitly state the solution to Harazišvili's problem on $\mathbb{Q}$-invariant extensions of $l_{1}$ (cf. [Ha, Problem 4; C, Problem 1]).

2.11. Corollary. There is no maximal $\mathbb{Q}$-invariant extension of the Lebesgue measure on $\mathbb{R}$.

The next and final result of this section specifies the amount of isometric invariance that can be required from a possible extension of $l_{n}$ to a universal measure on $\mathbb{R}^{n}$.

2.12. Theorem. Assume that $2^{\omega}$ is large. Then the following are equivalent:

(i) There exists a universal G-invariant extension of $l_{n}$.

(ii) $G$ is discrete.

Moreover, if $G$ is discrete, $m$ is a $G$-invariant extension of $l_{n}$ and there exists an extension of $m$ to a universal measure on $\mathbb{R}^{n}$, then there exists a G-invariant one.

Proof. (i) $\Rightarrow$ (ii) This follows from 2.7 and 2.9.

(ii) $\Rightarrow$ (i) It is well known that if $2^{\omega}$ is large, then there exists an extension of $l_{n}$ to a universal measure on $\mathbb{R}^{n}$. Hence it is enough to prove the last part of the theorem.

So let $m: \mathfrak{M} \rightarrow[0, \infty]$ be a $G$-invariant extension of $l_{n}$ which can be extended to a universal measure $\mu$ on $\mathbb{R}^{n}$. Proceed as in the proof of 2.10 , taking $\mathfrak{M}_{2}=\mathscr{P}(S)$ and $m_{2}=\mu \mid \mathscr{P}(S)$.

The above theorem should be compared with the well-known results on $G$ invariant extensions of $l_{n}$ to universal finitely-additive measures on $\mathbb{R}^{n}$. Such an extension exists if and only if $G$ is a so-called amenable group (see [Wag, Theorem 11.20]). For example, $G$ can be $D_{n}$ for $n=1$ or $n=2$ but it cannot be $D_{n}$ for $n>2$ by the famous Banach-Tarski paradox (see [Wag]).

2.13. Remark. (i) It is not difficult to strengthen 2.8(i) as follows: $G$ is discrete if and only if every $G$-orbit is discrete.

(ii) By a result of Kunen (see $[\mathrm{Ku}]$ ), there is an extension of $l_{n}$ which cannot be extended to a universal measure on $\mathbb{R}^{n}$. Accordingly, we cannot strengthen 
the last part of 2.12 by claiming that under some conditions on $G$, every $G$ invariant extension of $l_{n}$ has a universal $G$-invariant extension (even assuming that $2^{\omega}$ is large).

\section{Generalizations}

There are two natural directions in which one may try to generalize the results of $\S 1$. The first is to consider groups of arbitrary size, i.e., not necessarily of countable cardinality. It must be repeated that the general case of the invariant extension problem remains unsolved. Nevertheless, it seems worthwhile to realize that the arguments leading to 1.6 can be easily adapted to establish the following more general fact:

3.1. Theorem. Let $G$ be a group of bijections of a set $X$. Suppose that there exists a group $H$ of bijections of $X$ such that $\{h \mid G x: h \in H\}=\{g \mid G x: g \in G\}$ and $|H|<\kappa$, for every $x \in X$ where $\kappa$ is an uncountable cardinal.

Then the following are equivalent:

(i) Every $\sigma$-finite (finite) nonuniversal $\kappa$-additive $G$-invariant measure on $X$ has a proper $\kappa$-additive $G$-invariant extension.

(ii) The set $\{x \in X$ : there exists a (finite) nontrivial minimal G-partition of $G x\}$ is small.

The other direction of possible generalizations is to consider a wider class of measures. Semiregularity seems to be a natural assumption for which we may hope to obtain a different answer to the extension problem. The examination of the reasoning from $\S 1$ shows that, actually, for at most countable groups the answer is just the same as in the $\sigma$-finite case.

3.2. Proposition. Let $G$ be an at most countable group of bijections of $X$. Then the following are equivalent:

(i) Every semiregular, nonuniversal, G-invariant measure on $X$ has a proper, semiregular, G-invariant extension.

(ii) Every $\sigma$-finite, nonuniversal, $G$-invariant measure on $X$ has a proper $G$-invariant extension.

The methods developed in $\S 2$ to deal with invariant extensions of the Lebesgue measure can be applied to some other cases of interest. In particular we can use essentially the same proofs in order to establish the analogue of 2.10 and 2.12 obtained by replacing $\mathbb{R}^{n}$ by the $n$-dimensional unit sphere $S^{n}$ in $\mathbb{R}^{n+1}, D_{n}$ by the group of isometries of $S^{n}$, and $l_{n}$ by the Lebesgue measure on $S^{n}$.

By minor modifications of arguments we can also obtain:

3.3. Theorem. Let $X$ be an uncountable locally compact Polish (i.e., separable and complete) metric group and let $G$ be an at most countable subgroup of $X$. Then:

(i) If $G$ is dense in $X$, then every $G$-invariant extension of the Haar measure $\lambda$ on $X$ has a proper $G$-invariant extension. 
(ii) If $G$ is discrete, then every nonuniversal G-invariant extension of $\lambda$ has a proper G-invariant extension.

Proof. (i) Apply 1.12 using the fact that there are open subsets of $G$ of arbitrary small positive Haar measure.

(ii) Proceed as in the proof of 2.10. Notice that Kuratowski-Maitra's theorem works in this case (see $[\mathrm{KM}]$ ).

3.4. Corollary. Assume that $2^{\omega}$ is large. Let $X$ be an uncountable locally compact Polish metric group and let $G$ be an arbitrary subgroup of $X$. Then the following are equivalent:

(i) There exists a universal G-invariant extension of the Haar measure $\lambda$ on $X$.

(ii) $G$ is discrete.

Proof. (i) $\Rightarrow$ (ii) We have $|G| \leq \omega$ since otherwise, by a theorem of RyllNardzewski and Telgársky [RNT], there would be no universal $G$-invariant measure on $X$. Now use 3.3(i).

(ii) $\Rightarrow$ (i) Proceed as in the proof of part (ii) of 3.3. Use the well-known fact that if $2^{\omega}$ is large, then $\lambda$, being a Borel measure on a Polish space, can be extended to a universal measure on $X$.

\section{REFERENCES}

[BH] R. G. Burns and V. W. Hale, A note on group rings of certain torsion-free groups, Canad. Math. Bull. 15 (1972), 441-445.

[C] K. Ciesielski, Algebraically invariant extensions of $\sigma$-finite measures on Euclidean spaces, Trans. Amer. Math. Soc. 318 (1990), 261-273.

[CP] K. Ciesielski and A. Pelc, Extensions of invariant measures on Euclidean spaces, Fund. Math. 125 (1985), 1-10.

[F] D. R. Farkas, Crystallographic groups and their mathematics, Rocky Mountain J. Math. 11 (1981), 511-551.

[Ha] A. B. Harazišvili, Invariant extensions of the Lebesgue measure, Tbilisi Univ. Press, Tbilisi, 1983. (Russian)

[HR] E. Hewitt and K. A. Ross, Abstract harmonic analysis, Vol. I, Grundlehren Math. Wiss. vol. 115, Springer-Verlag, Berlin, 1979.

[Hu] A. Hulanicki, Invariant extensions of the Lebesgue measure, Fund. Math. 51 (1962), 111115.

[KaMe] M. I. Kargapolov and Ju. I. Merzljakov, Fundamentals of the theory of groups, Graduate Texts in Math., Springer-Verlag, Berlin-Heidelberg-New York, 1979.

[Ku] K. Kunen, Ph.D. thesis.

[KM] K. Kuratowski and A. Maitra, Some theorems on selectors and their applications to semicontinuous decomposition, Bull. Acad. Polon. Sci. Sér. Sci. Math. Astronom. Phys. 22 (1974), 877-882.

[LM] J. Loś and E. Marczewski, Extensions of measure, Fund. Math. 36 (1949), 267-276.

[P] A. Pelc, Invariant measures and ideals on discrete groups, Dissertationes Math. (Rozprawy Mat.) 255 (1986).

[RNT] C. Ryll-Nardzewski and R. Telgársky, The nonexistence of universal invariant measures, Proc. Amer. Math. Soc. 69 (1978), 240-242. 
[St] A. Strojnowski, On residually finite groups of isometries of $\mathbf{R}^{n}$ and extensions of measures, (to appear).

[Sz] E. Szpilrajn, Sur l'extension de la mesure lebesguienne, Fund. Math. 25 (1935), 551-558.

[Wag] S. Wagon, The Banach-Tarski paradox, Cambridge Univ. Press, Cambridge, 1985.

[War] F. W. Warner, Foundations of differentiable manifolds and Lie groups, Springer-Verlag, 1983.

[Z1] P. Zakrzewski, The existence of universal invariant measures on large sets, Fund. Math. 133 (1989), 113-124.

[Z2] __ Extensions of isometrically invariant measures on Euclidean spaces, Proc. Amer. Math. Soc. 110 (1990), 325-331.

Institute of Mathematics, University of Warsaw, PKiN 9P., 00-901 Warsaw, Poland 